\title{
Diagnosis and Management of Adverse Food Reactions
}

\author{
W Jean Dodds* \\ Hemopet, 938, California, USA \\ Received: March 07, 2018; Published: March 19, 2018 \\ *Corresponding author: W Jean Dodds, Hemopet, 938, California, USA, Tel: 310-828-4804; Email: info@hemopet.org
}

\section{Introduction}

Adverse food reactions in people and pets closely mimic those from environmental allergen and contact exposures, and are manifested typically as cutaneous or gastrointestinal signs or both [1-6]. Neurological sings including memory loss, confusion and cognitive dysfunction may also occur, especially in the aged. Some experts equate the increase in food-related disorders in pets with the promotion of dry kibble and wet canned foods by the commercial pet food industry, to address the time-strapped societal demands of our fast-paced Western world [6]. The question arises, however, whether modern domestic dogs and cats can adequately digest and assimilate dry commercial pet foods, when they are ancestrally carnivorous. Although cats have maintained their carnivorous nature over decades, dogs have evolved with domestication by adapting genomically a starch-rich diet [7]. This evolutionary adaptation of canines from the carnivorous wolf targeted 19 of the 36 genomic regions which contained genes important in brain function and behavior, and ten genes with key roles in starch digestion and fat metabolism [7].

This situation is confounded by the fact that many commercial pet foods contain meat and flavorings not listed or specified on the label. For example, a study in 2013 revealed that the actual ingredients matched those on the label in only two of twelve commercial diets analyzed [8]. For the other ten foods, bone fragments were detected from avian fragments in six of ten samples, fish in five of ten, and mammalian fragments in four of ten [8]. The conclusion of this study was that a novel protein homemade diet trial should be performed, if the dog is unresponsive to a commercial regimen, before one can truly rule out a food allergen component. In a similar earlier study, three of four commercial dry foods containing venison tested positive for soy and one food tested positive for beef, even though the label specified a diet limited to venison [9]. In this situation, using venison-based dry foods for food elimination trials did not prove to be reliable. Allergens in beef, cow's milk and lamb are often believed to be the culprit(s) in cutaneous adverse food reactions [3,10-13]. Other common canine food allergens for dogs were: chicken, chicken eggs, wheat, soy and corn, while cats commonly reacted to chicken, fish and dairy products [10].
Bovine immunoglobulin G (IgG) was found to be the common allergen in cow's milk, and a specific phosphoglucomutase and heavy chain IgG were the responsible allergens in extracts of both lamb and beef [13]. Another study examined 14 dogs with clinical hypersensitivity to soy and corn [14]. Minimal clinical signs of pruritus were seen once they were fed a limited antigen diet of duck and rice. Following sequential re-challenge with cornstarch, corn, soy and a hydrolyseddiet, however, significant pruritus returned with cornstarch, corn and soy but not with the hydrolysed diet [14]. A later study from 2016, found the most commonly encountered food allergens in dogs were beef, dairy products, chicken and wheat. In cats, beef, fish and chicken were the most common food allergens [3]. An intriguing finding for beef in the most recent study from Germany and France, even though no positive salivary reactions to beef were found, all of these dogs reacted to corn, a grain commonly fed to beef cattle in this area of Europe and found in their flesh [15].

Another recent study [16], Presented data from healthy dogs comparing two commonly used commercial serum tests for food reactivity and the saliva-based test [6], Thirty dogs ranging from one to 10 years of age (median $=4$ years) and weighing between 2.2 and $50.8 \mathrm{~kg}$ (median $=20 \mathrm{~kg}$ ) were studied from the hospital population at a veterinary teaching institution. All 30 asymptomatic dogs had at least one positive response to a food, which was not unexpected. One or more dogs also tested positive to most [12]. Or all of the 14 foods evaluated. Interestingly, there was no predictable concordance between positive responses and historical food exposure. The authors concluded that over-diagnosis of foodrelated allergens could result if these assays were used instead of performing strict elimination diet trials [16]. These conclusions, however, related only to healthy dogs, as no dogs suspected of or proven to have diet-related adverse events were studied.

The German and French study showed variable sensitivity and specificity with respect to the saliva-based results obtained for IgA and IgM reactivates to the foods tested, and some of these German patients were not available for follow up. Further assessment of these findings was compromised as the study control group of healthy dogs came from a commercial pet food company in France. These dogs ate diets containing chicken, beef, corn, wheat and rice 
made by the company and changed regularly for the purpose of palatability testing; and so dietary immune tolerance could have been created by the rotational nature of their diet. A preferred control group of dogs would have been healthy pets coming to the German university veterinary clinic hospital for wellness checkups. Collectively, these results provide a plausible explanation of why pets with suspected or even proven adverse food reactions may fail to respond to commercial limited antigen source foods $[6,14,17]$. Lastly, the data summarized above are further confounded by the fact that many pets also receive a variety of supplements, preventive pharmaceuticals such as those for heartworm, flea and tick exposures, as well as puppy and periodic booster vaccines.

These products usually contain meat, especially beef, pork and chicken, as well as other flavorings and several types of fish oils and nearly all vaccines contain fetal calf serum [18]. The problem is more complicated when veterinary therapeutic and supplement items and over-the-counter products may not accurately list the ingredients or their antigen sources on the label or product insert [17]. Thus, when recommending food elimination trials, only nonflavored oral or topical therapies, pill pockets, and supplements should be used. Gelatin capsules may contain either beef or pork proteins and also should not be administered during these trials.

\section{References}

1. Olivry T, Deboer DJ, Prélaud P, Bensignor E (2007) International Task Force on Canine Atopic Dermatitis. Food for thought: pondering the relationship between canine atopic dermatitis and cutaneous adverse food reactions. Vet Dermatol 18(6): 390-391.

2. Gaschen FP, Merchant SR (2011) Adverse food reactions in dogs and cats. Vet Clin North Am Small Anim Pract 41(2): 361-379.

3. Mueller RS, Olivry T, Prélaud P (2016) Critically appraised topic on adverse food reactions of companion animals (2): common food allergen sources in dogs and cats. BMC Vet Res 12: 9.

4. Olivry T, Mueller RS (2017) Critically appraised topic on adverse food reactions of companion animals (3): prevalence of cutaneous adverse food reactions in dogs and cats. BMC Vet Res 13: 51.
5. Verlinden A, Hesta M, Millet S, Janssens GP (2006) Food allergy in dogs and cats: a review. Clin Rev Food Sci Nutr 46: 259-273.

6. Dodds WJ (2017) Diagnosis of canine food sensitivity and intolerance using saliva: report of outcomes. J Am Hol Vet Med Assoc 49: 32-43.

7. Axelsson E, Ratnakumar A, Arendt MJ, Maqbool K, Webster MT, et al. (2003) The genomic signature of dog domestication reveals adaptation to a starch-rich diet. Nature 495: 360-364.

8. Ricci R, Granato A, Vascellari M, Boscarato M, Palagiano C, et al. (2013) Identification of undeclared sources of animal origin in canine dry foods used in dietary elimination trials. J Anim Physiol Anim Nutr 97: 32-38.

9. Raditic DM, Remillard RL, Tater KC (2011) ELISA testing for common food antigens in four dry dog foods used in dietary elimination trials. J Anim Physiol Anim Nutr 95: 90-97.

10. Lewis T (2017) Diagnosing food allergies in dogs and cats - bring your case to trial. DVM360 Magazine, USA, p. 5.

11. Carlotti DN (2014) cutaneous manifestations of food hypersensitivity. In Veterinary Allergy, Chichester, Wiley Blackwell, UK, pp. 108-114.

12. Olivry T, Bizikova P (2010) A systematic review of the evidence of reduced antigenicity and clinical benefit of food hydrolysates in dogs with cutaneous adverse food reactions. Vet Dermatol 21: 32-41.

13. Martin A, Sierra MP, González JL, Arévalo MA (2004) Identification of allergens responsible for canine cutaneous adverse food reactions to lamb, beef and cow's milk. Vet Dermatol 15: 349-356.

14. Jackson HA, Jackson MW, Coblentz, L, Hammerberg B (2003) Evaluation of the clinical and allergen specific serum immunoglobulin E responses to oral challenge with cornstarch, corn, soy and a soy hydrolysate diet in dogs with spontaneous food allergy. Vet Dermatol 14: 181-187.

15. Udraite Vovk L, Watson A, Dodds WJ, Klinger CJ, Classen J, et al. (2018) Testing for food-specific antibodies in saliva and blood of food-allergic and healthy dogs.

16. Lam ATH, Johnson LN, Heinze CR (2017) Evaluation of clinical accuracy of serological and salivary testing for food allergens in asymptomatic dogs. NAVDF Proc p. 26-29.

17. Parr JM, Remillard RL (2014) Common cofounders of dietary elimination trials contain the antigens soy, pork, and beef. I Am An Hosp Assoc 50(5): 298-304.

18. Dodds WJ (2016) Adjuvants and additives in human and animal vaccines. Med Res Archives 2(5): 1-8.
cC) (i) This work is licensed under Creative

Submission Link: https://biomedres.us/submit-manuscript.php

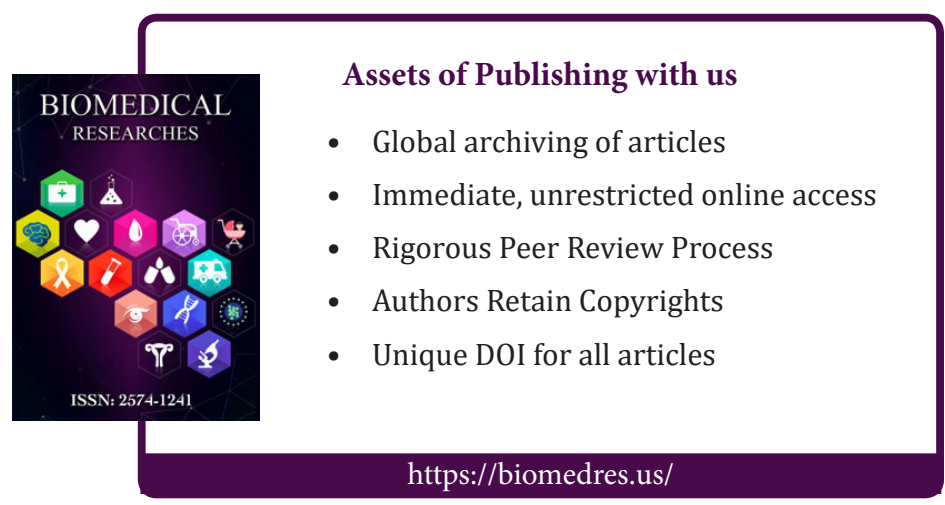

